\title{
Longitudinal Study of Gray Matter Changes in Parkinson Disease
}

\author{
X. Jia, P. Liang, Y. Li, L. Shi, D. Wang, and K. Li
}

\section{ABSTRACT}

BACKGROUND AND PURPOSE: The pathology of Parkinson disease leads to morphological brain volume changes. So far, the progressive gray matter volume change across time specific to patients with Parkinson disease compared controls remains unclear. Our aim was to investigate the pattern of gray matter changes in patients with Parkinson disease and to explore the progressive gray matter volume change specific to patients with Parkinson disease with disease progression by using voxel-based morphometry analysis.

MATERIALS AND METHODS: Longitudinal cognitive assessment and structural MR imaging of 89 patients with Parkinson disease (62 men) and 55 healthy controls ( 33 men) were from the Parkinson's Progression Markers Initiative data base, including the initial baseline and 12-month follow-up data. Two-way analysis of covariance was performed with covariates of age, sex, years of education, imaging data from multiple centers, and total intracranial volume by using Diffeomorphic Anatomical Registration Through Exponentiated Lie Algebra tool from SPM8 software.

RESULTS: Gray matter volume changes for patients with Parkinson disease were detected with decreased gray matter volume in the frontotemporoparietal areas and the bilateral caudate, with increased gray matter volume in the bilateral limbic/paralimbic areas, medial globus pallidus/putamen, and the right occipital cortex compared with healthy controls. Progressive gray matter volume decrease in the bilateral caudate was found for both patients with Parkinson disease and healthy controls, and this caudate volume was positively associated with cognitive ability for both groups. The progressive gray matter volume increase specific to the patients with Parkinson disease was identified close to the left ventral lateral nucleus of thalamus, and a positive relationship was found between the thalamic volume and the tremor scores in a subgroup with tremor-dominant patients with Parkinson disease.

CONCLUSIONS: The observed progressive changes in gray matter volume in Parkinson disease may provide new insights into the neurodegenerative process. The current findings suggest that the caudate volume loss may contribute to cognitive decline in patients with Parkinson disease and the progressive thalamus enlargement may have relevance to tremor severity in Parkinson disease.

ABBREVIATIONS: $B A=$ Brodmann area; Control $1=$ control baseline; Control $2=$ control follow-up; DARTEL $=$ Diffeomorphic Anatomical Registration Through Exponentiated Lie Algebra Toolbox; GMV = gray matter volume; MDS-UPDRS III = Movement Disorder Society-Unified Parkinson's Disease Rating Scale III; MNI = Montreal Neurological Institute; MoCA = Montreal Cognitive Assessment; PD = Parkinson disease; PD1 = PD baseline; PD2 = PD follow-up; VBM = voxel-based morphometry

$\mathrm{P}$ arkinson disease $(\mathrm{PD})$ is a progressive neurodegenerative disorder characterized by the degeneration of dopamine neurons in the substantia nigra, with other neurons in the

Received June 27, 2014; accepted after revision April 20, 2015

From the Department of Radiology (X.J., P.L., Y.L., K.L.), Xuanwu Hospital, Capital Medical University, Beijing, China; Beijing Key Laboratory of MRI and Brain Informatics (X.J., P.L., Y.L., K.L.), Beijing, China; and Department of Imaging and Interventional Radiology (L.S., D.W.), The Chinese University of Hong Kong, Shatin, New Territories, Hong Kong SAR, China.

The Parkinson's Progression Markers Initiative is funded by the Michael J. Fox Foundation for Parkinson's Research and funding partners, including Abbott, Avid, Biogen Idec, Bristol-Myers Squibb, Covance, Elan, GE Healthcare, Genentech, GlaxoSmithKline, Lilly, Merck, Meso Scale Discovery, Pfizer, Roche, and Union chimique belge (details of the Parkinson's Progression Markers Initiative funding partners is found at http://www.ppmi-info.org/about-ppmi/who-we-are/study-sponsors/). This work was supported by a grant from the National Natural Science Foundation cortex and subcortical nuclei also affected during the course of the disease. This pathology might lead to morphologic brain changes.

Voxel-based morphometry (VBM) analysis has been used to

of China (61105118, 31400958, 61473196), the Beijing Nova Program (No. Z12111000250000, Z131107000413120), the Key Projects in the National Science and Technology Pillar Program during the Twelfth Five-Year Plan Period (2012BAl10B04), and the Open Research Fund of the State Key Laboratory of Cognitive Neuroscience and Learning (CNLZD1302).

Please address correspondence to Kuncheng Li, MD, Xuanwu Hospital, Capital Medical University, 45 Chang Chun St, Xi Cheng District, Beijing 100053, China; e-mail: cjr.likuncheng@vip.163.com

- Indicates open access to non-subscribers at www.ajnr.org

5 Indicates article with supplemental on-line photos.

http://dx.doi.org/10.3174/ajnr.A4447 
assess the cortical gray matter changes in patients with PD. Some cross-sectional studies were performed to compare the differences between patients with PD and healthy controls. However, these PD-VBM studies have not yet drawn any congruent conclusions. Some studies have shown distributed brain atrophy in cortical and subcortical regions, including the frontal lobe, temporal lobe, parietal lobe, occipital lobe, and the limbic/paralimbic areas. ${ }^{1-8}$ On the other hand, 1 study reported an increase of GM in the thalamus in patients with PD with unilateral resting tremor compared with controls. ${ }^{9}$ A recent study has observed not only brain volume loss in the occipital region but also volume increase in the limbic/paralimbic system. ${ }^{10}$ In addition, some studies have failed to find any GM change. ${ }^{11-14}$ In fact, few of these previous findings were wholly consistent with each other. These inconsistencies may result from the patient heterogeneity, such as the age, disease duration, disease severity, and variable covariates used in VBM analysis, which may confound the effect of results in between-group differences. Therefore, this issue of brain volume change in PD groups required further examination.

To our knowledge, few studies have focused on the progression of regional volume changes in PD by using VBM. One longitudinal study showed a progressive gray matter volume (GMV) decrease in patients with PD with and without dementia with disease progression during a mean follow-up period of 25 months. ${ }^{15}$ In that study, a progressive GMV decrease in the limbic/paralimbic and temporo-occipital regions was observed in patients with $\mathrm{PD}$, while in patients with dementia, the loss mainly involved the neocortical regions. However, in that study, no healthy matched controls were included. So far, the progressive GMV change across time specific to patients with PD compared with controls remains unclear.

Thus, the main goals of the present study were to examine the GMV change in the PD group compared with healthy controls and to explore the progressive GMV change specific to patients with PD compared with controls with disease progression.

\section{MATERIALS AND METHODS Data Acquisition Centers}

In the present study, the data were selected from the following 7 MR imaging centers: Emory University, Atlanta, Georgia; Johns Hopkins University, Baltimore, Maryland; Northwestern University, Chicago, Illinois; Baylor College of Medicine, Houston, Texas; Universitat Tubingen, DZNE und Hertie-Institut fur Klinische Hirnforschung, Tubingen, Germany; Paracelsus-Elena Clinic Kassel/University of Marburg, Kassel, Germany; and Cleveland Clinic Foundation, Cleveland, Ohio.

\section{Subjects}

Data used in this article were from the Parkinson's Progression Markers Initiative data base (http://www.ppmi-info.org/data). This is the first large-scale, comprehensive, observational, international, multicenter study to identify PD progression biomarkers to improve the understanding of the disease etiology and the effectiveness of disease-modifying therapeutic trials. ${ }^{16}$ Inclusion criteria of patients with PD for multiple centers were the follow- ing: 1) at least 2 of the following: resting tremor, bradykinesia, and rigidity (must have either resting tremor or bradykinesia) or either asymmetric resting tremor or asymmetric bradykinesia; 2) Hoehn and Yahr stage 1 or 2 at baseline; 3 ) dopamine transporter SPECT scan showing a dopamine transporter deficit; 4) not expected to require PD medication within at least 6 months from baseline; and 5) 30 years of age or older. Exclusion criteria for patients with $\mathrm{PD}$ were the following: 1) currently taking levodopa, dopamine agonists, monoamine oxidase inhibitors- $\mathrm{B}$, amantadine, or other PD medications or taking them within 60 days of baseline; and 2) use of investigational drugs or devices within 60 days before baseline.

Inclusion criterion for healthy controls was 30 years of age or older. Exclusions were the following: 1) current or active clinically significant neurologic disorder; 2) first-degree relative with idiopathic PD; 3) Montreal Cognitive Assessment (MoCA) score of $\leq 26$; and 4) use of investigational drugs or devices within 60 days before baseline.

In the present study, all subjects were selected on the basis of the following criteria: 1) Participants did not have depression, with a Geriatric Depression Scale score of $<5,{ }^{17}$ or dementia, which was determined by the following: A) cognitive function that is impaired in $>1$ cognitive domain, B) decline from premorbid function, and C) significant impact of cognitive impairment on daily function; ${ }^{16}$ 2) Subjects were studied 2 times (ie, baseline and 1-year follow-up) by September 12, 2013, and with the same scanning parameters; and 3) all MR imaging data were obtained on a Tim Trio 3T scanner (Siemens, Erlangen, Germany). There were 89 patients with $\mathrm{PD}$ (62 men, $62.0 \pm 8.7$ years of age) and 55 healthy controls ( 33 men, $58.4 \pm 11.1$ years of age). Table 1 shows the demographic and clinical data of the subjects.

\section{Clinical Assessment}

For patients with $\mathrm{PD}$, the disease stage was scored by using the Hoehn and Yahr stage score, and the disease severity, by using the Movement Disorder Society-Unified Parkinson's Disease Rating Scale III (MDS-UPDRS III). In addition, all subjects were administered the University of Pennsylvania Smell Identification Test for assessment of olfactory function and Scales for Outcomes in Parkinson's Disease-Autonomic for assessment of autonomic disorder. To evaluate the neuropsychological state, we administered the following to all the subjects: the Benton Judgment of Line Orientation Score, the Geriatric Depression Scale, the MoCA, the Semantic Fluency total score, the Hopkins Verbal Learning Test, the Modified Schwab and England Activities of Daily Living, and the Symbol Digit Modalities score.

\section{MR Imaging Data Acquisition}

MR imaging data acquisition was performed on a Tim Trio 3T scanner (Siemens). High-resolution structural images were collected by using a 3D magnetization-prepared rapid acquisition of gradient echo T1-weighted sequence with the following parameters: sagittal section thickness, $1.0 \mathrm{~mm}$; no gap; TR, $2300 \mathrm{~ms}$; TE, $2.98 \mathrm{~ms}$; flip angle, $9^{\circ}$; FOV , $240 \times 256 \mathrm{~mm}$; matrix size, $240 \times$ 256; TI, $900 \mathrm{~ms}$; voxel size, $1 \times 1 \times 1 \mathrm{~mm}^{3}$. The image plane aligned in the sagittal plane along the hemispheric fissure and axially along the anterior/posterior commissure plane. The 176 
Table 1: Clinical results at baseline and follow-up in PD and control samples ${ }^{a}$

\begin{tabular}{|c|c|c|c|c|c|c|}
\hline & \multicolumn{3}{|c|}{ Baseline } & \multicolumn{3}{|c|}{ Follow-Up } \\
\hline & $\operatorname{PD}(n=89)$ & Control $(n=55)$ & $P$ Value $^{\text {b }}$ & $\operatorname{PD}(n=89)$ & Control $(n=55)$ & $P$ Value \\
\hline Age (yr) & $62.0 \pm 8.7$ & $58.4 \pm 11.1$ & $<.05$ & $63.0 \pm 8.7$ & $59.4 \pm 11.1$ & $<.05$ \\
\hline Sex (male/female) & $62 / 27$ & $33 / 22$ & .234 & $62 / 27$ & $33 / 22$ & .234 \\
\hline Education (yr) & $15.2 \pm 2.9$ & $15.4 \pm 2.9$ & .62 & $15.2 \pm 2.9$ & $15.4 \pm 2.9$ & .62 \\
\hline Total intracranial volume $(\mathrm{mL})$ & $1570.2 \pm 143.5$ & $1,505.9 \pm 142.3$ & .01 & $1,560.2 \pm 143.1$ & $1,515.3 \pm 140.9$ & .057 \\
\hline Hoehn and Yahr stage & $1.6 \pm 0.5$ & - & - & $1.8 \pm 0.5$ & - & - \\
\hline MDS-UPDRS Part III ${ }^{\mathrm{C}}$ & $21.9 \pm 9.1$ & $0.55 \pm 1.33$ & $<.001$ & $23.0 \pm 10.0$ & $0.82 \pm 1.44$ & $<.001$ \\
\hline $\begin{array}{l}\text { Dose of levodopa-equivalent medication } \\
(\mathrm{mg} / \text { day })^{d}\end{array}$ & - & - & - & $409.8 \pm 369.3$ & - & - \\
\hline Benton Judgment of Line Orientation score & $12.7 \pm 2.2$ & $13.3 \pm 1.8$ & .099 & $12.53 \pm 2.2$ & $12.6 \pm 2.5$ & .146 \\
\hline Geriatric Depression Scale & $2.7 \pm 2.9$ & $1.5 \pm 3.0$ & $<.05$ & $2.8 \pm 3.0$ & $1.4 \pm 2.5$ & $<.05$ \\
\hline Montreal Cognitive Assessment ${ }^{\mathrm{e}}$ & $27.5 \pm 2.0$ & $28.4 \pm 1.2$ & .001 & $26.2 \pm 2.9$ & $27.6 \pm 2.0$ & 0.002 \\
\hline Semantic Fluency total score & $48.7 \pm 9.8$ & $52.4 \pm 11.6$ & $<.05$ & $48.2 \pm 10.3$ & $53.7 \pm 11.6$ & $<.05$ \\
\hline HVLT Delayed Recognition False Alarms & $1.5 \pm 1.5$ & $1.5 \pm 1.9$ & .958 & $1.3 \pm 1.3$ & $1.3 \pm 1.7$ & .943 \\
\hline HVLT Delayed Recognition hits & $11.5 \pm 0.8$ & $11.2 \pm 1.0$ & $<.05$ & $11.5 \pm 0.8$ & $11.0 \pm 1.6$ & $<.05$ \\
\hline HVLT Immediate Recall & $26.4 \pm 4.7$ & $24.3 \pm 5.4$ & $<.05$ & $27.2 \pm 4.4$ & $23.3 \pm 6.1$ & $<.001$ \\
\hline Modified Schwab and England ADL & $94.7 \pm 5.4$ & - & - & $91.8 \pm 6.8$ & - & - \\
\hline Symbol Digit Modalities score & $40.9 \pm 9.9$ & $48.9 \pm 11.20$ & $<.001$ & $39.6 \pm 11.2$ & $47.1 \pm 10.0$ & $<.001$ \\
\hline UPSIT & $21.1 \pm 9.0$ & $33.2 \pm 4.9$ & $<.001$ & - & - & - \\
\hline SCOPA-AUT & $9.2 \pm 6.4$ & $6.9 \pm 4.4$ & $<.001$ & $11.9 \pm 6.4$ & $7.6 \pm 5.8$ & $<.001$ \\
\hline
\end{tabular}

Note:-MDS-UPDRS indicates Movement Disorder Society-Unified Parkinson's Disease Rating Scale; HVLT, Hopkins Verbal Learning Test; ADL, Activities of Daily Living; UPSIT, University of Pennsylvania Smell Identification Test; SCOPA-AUT, Scales for Outcomes in Parkinson's Disease-Autonomic; -, no value.

${ }^{a}$ Values are expressed as mean (SD).

b $P$ values were derived from the Student $t$-test comparing the 2 groups except for "Sex," which was derived using the $\chi^{2}$ test.

'MDS-UPDRS III was performed in the off-state at follow-up.

${ }^{\mathrm{d}}$ There were $83.15 \%$ of patients with PD who were on dopaminergic therapy at 1-year follow-up.

e Significant score decrease in the follow-up both for patients with PD and healthy controls compared with baseline $(P<.05)$.

sections covered the entire brain from ear to ear and the bottom of the cerebellum to the vertex.

\section{VBM-Diffeomorphic Anatomical Registration Through Exponentiated Lie Algebra Analysis}

Voxel-based morphometry was performed by using SPM8 (http://www.fil.ion.ucl.ac.uk/spm/software/spm8) and the Diffeomorphic Anatomical Registration Through Exponentiated Lie Algebra Toolbox (DARTEL, part of SPM) registration method. ${ }^{18}$ The VBM preprocessing included 5 steps: 1) T1-weighted images were segmented by using the standard unified segmentation model in SPM8 to produce gray matter, white matter, and CSF probability maps in the Montreal Neurological Institute (MNI) space; 2 ) the study-specific GM templates were created from the entire image dataset by using the DARTEL technique; 3) an initial affine registration of the GM templates to the tissue probability maps in MNI space was performed; 4) nonlinear warping of GM images to the GM template in MNI space was performed and then was used in the modulation step to ensure that the relative volumes of GM were preserved following the spatial normalization procedure by the Jacobian determinant of the deformation field to adjust volume changes; ${ }^{19}$ and 5) The modulated, normalized GM images (representing GMV; voxel size, $1.5 \times 1.5 \times 1.5 \mathrm{~mm}^{3}$ ) were smoothed with a $6-\mathrm{mm}$ full width at half maximum isotropic Gaussian kernel. The total intracranial volume was represented by the sum of the GM, WM, and CSF volumes. In addition, in the VBM-DARTEL analysis, checking of sample homogeneity was performed in SPM8 on the normalized data for quality control.

\section{Statistical Analysis}

Demographic data and neuropsychological measures at baseline were analyzed by SPSS, Version 19 (IBM, Armonk, New York), with the Student $t$ test for continuous variables and the $\chi^{2}$ test for dichotomous variables.

The present study was organized into 2 [(time: 12-month follow-up versus baseline) $\times 2$ (group: $\mathrm{PD}$ versus control)] flexible factorial designs. We thus had 4 conditions: PD baseline (PD1), PD follow-up (PD2), control baseline (Control1), and control follow-up (Control2). Age, sex, years of education, imaging data from multiple centers, and total intracranial volume were entered as regressors into the flexible factorial design to establish the regional GM volume changes.

The main effect of "group" was revealed by the following 2 contrasts: The decreased GM volumes for patients with PD were determined by the contrast of $[($ Control $1+$ Control 2$)>(\mathrm{PD} 1+$ $\mathrm{PD} 2)$ ], and the increased GM volumes for patients with PD were determined by the contrast of $[(\mathrm{PD} 1+\mathrm{PD} 2)>($ Control $1+$ Contol2)].

The main effect of "time" was revealed by the following 2 contrasts: The decreased GM volumes for follow-up were determined by the contrast of $[(\mathrm{PD} 1+$ Control1 $)>(\mathrm{PD} 2+$ Control 2$)]$, and the increased GM volumes for follow-up were determined by the contrast of $[(\mathrm{PD} 2+$ Control2 $)>(\mathrm{PD} 1+$ Control1 $)]$. In addition, 2 simple main effects of $(\mathrm{PD} 1>\mathrm{PD} 2)$ and (Control1 $>$ Control2) were performed.

The interaction effect of "group" by "time" was revealed by the following 2 contrasts: The increased GM volumes specific to patients with PD with time was determined by the contrast of $[(\mathrm{PD} 2>\mathrm{PD} 1)>($ Control $2>$ Control1 $)]$, and the decreased GM volumes specific to patients with $\mathrm{PD}$ with time were determined by the contrast of $[(\mathrm{PD} 1>\mathrm{PD} 2)>$ (Control1 > Control2)].

The statistical significance threshold was set at $P<.001$ cor- 
Table 2: GMV changes for patients with PD compared with controls

\begin{tabular}{|c|c|c|c|c|c|c|}
\hline \multirow[b]{2}{*}{ Regions } & \multirow[b]{2}{*}{ BA } & \multirow[b]{2}{*}{ Cluster Size (Voxel) } & \multicolumn{3}{|c|}{ MNI } & \multirow[b]{2}{*}{$T$ Score } \\
\hline & & & $x$ & $\mathbf{Y}$ & Z & \\
\hline \multicolumn{7}{|l|}{ Control > PD } \\
\hline Rt. superior frontal gyrus & 9 & 262 & 24 & 56 & 40 & 17.19 \\
\hline Rt. superior frontal gyrus & 9 & & 18 & 60 & 36 & 8.52 \\
\hline Rt. middle frontal gyrus & 10 & & 24 & 63 & 27 & 7.00 \\
\hline Rt. middle frontal gyrus & 9 & 466 & 39 & 42 & 42 & 11.48 \\
\hline Rt. middle frontal gyrus & 10 & & 39 & 62 & 16 & 10.62 \\
\hline Rt. superior frontal gyrus & 9 & & 36 & 53 & 31 & 9.84 \\
\hline Lt. superior frontal gyrus & 9 & 790 & -18 & 59 & 34 & 13.37 \\
\hline Lt. superior frontal gyrus & 8 & & -26 & 44 & 48 & 13.05 \\
\hline Lt. superior frontal gyrus & 9 & & -23 & 53 & 42 & 12.68 \\
\hline Lt. inferior temporal gyrus & 37 & 813 & -65 & -60 & -8 & 10.78 \\
\hline Lt. middle temporal gyrus & 21 & & -65 & -62 & 6 & 10.15 \\
\hline Lt. inferior temporal gyrus & 20 & & -63 & -54 & -20 & 10.10 \\
\hline Rt. superior parietal lobule & 7 & 225 & 45 & -69 & 48 & 8.55 \\
\hline Rt. inferior parietal lobule & 40 & & 53 & -61 & 48 & 7.75 \\
\hline Rt. angular gyrus & 39 & & 57 & -67 & 36 & 7.38 \\
\hline Lt. caudate body & & 459 & -9 & -1 & 21 & 13.90 \\
\hline Lt. caudate body & & & -6 & 3 & 15 & 13.24 \\
\hline Lt. caudate body & & & -12 & -9 & 22 & 11.04 \\
\hline Rt. caudate body & & 460 & 8 & 6 & 15 & 11.70 \\
\hline Rt. caudate body & & & 12 & -6 & 22 & 9.48 \\
\hline Rt. caudate body & & & 15 & -15 & 25 & 8.52 \\
\hline \multicolumn{7}{|l|}{ PD $>$ Control } \\
\hline Lt. inferior frontal gyrus & 47 & 109 & -15 & 24 & -15 & 10.63 \\
\hline Rt. superior frontal gyrus & 11 & 212 & 23 & 51 & -23 & 9.35 \\
\hline Rt. superior frontal gyrus & & & 24 & 60 & -21 & 8.63 \\
\hline Rt. superior frontal gyrus & & & 32 & 47 & -18 & 7.56 \\
\hline Rt. anterior cingulate cortex & 32 & 182 & 17 & 30 & 30 & 11.73 \\
\hline Rt. anterior cingulate cortex & & & 15 & 21 & 39 & 10.82 \\
\hline Rt. anterior cingulate cortex & & & 20 & 35 & 21 & 9.28 \\
\hline Rt. middle occipital gyrus & 19 & 124 & 42 & -90 & 18 & 7.89 \\
\hline Lt. globus pallidus internus & & 390 & -9 & 0 & -5 & 13.84 \\
\hline Lt. hippocampus & & & -30 & -19 & -9 & 9.78 \\
\hline Lt. globus pallidus internus & & & -14 & -6 & -8 & 8.47 \\
\hline Lt. subthalamic nucleus & & 113 & -11 & -12 & -5 & 11.80 \\
\hline Rt. globus pallidus internus & & 451 & 18 & -7 & -8 & 11.59 \\
\hline Rt. globus pallidus internus & & & 12 & 3 & -5 & 11.26 \\
\hline Rt. putamen & & & 30 & -18 & -8 & 10.97 \\
\hline
\end{tabular}

Note:-BA indicates Brodmann area; Rt., right; Lt., left.

rected for multiple comparisons (family-wise error) with a minimum cluster size of 100 contiguous voxels, but with an exception of 50 contiguous voxels for the interaction effect. The coordinates of voxels were transferred from Montreal Neurological Institute space to Talairach space by using $M$. Brett's transformation (http://imaging.mrc-cbu.cam.ac.uk/imaging/MniTalairach).

\section{ROI Analysis}

To examine the GMV change with time in the present study, we defined the ROIs by using 2 methods. On the one hand, those ROIs based on the results in the present study were defined by cluster-based method-that is, clusters that showed significant differences in the contrasts of $[(\mathrm{PD} 1+$ Control1 $)>(\mathrm{PD} 2+$ Control2)] (bilateral caudate body; MNI coordinates: $-8,3,18$; $9,5,18)$ and $[(\mathrm{PD} 2>\mathrm{PD} 1)>($ Control2 $>$ Control1 $)]$ (left thalamus; MNI coordinates: $-21,-24,3)$ were defined as ROIs by using xjView software (http://www.alivelearn.net/xjview). On the other hand, to compare with the previous longitudinal study, ${ }^{15}$ we defined additional ROIs on the basis of a sphere with a radius of $6 \mathrm{~mm}$ centered at the anterior cingulate cortex (MNI coordinates: 1, 31 19), posterior cingulate cortex (MNI coordinates: 11,
$-49,30$ ), hippocampus (MNI coordinates: $-22,-19,-11)$, and hypothalamus (MNI coordinates: $1,-9,-5)$ by using WFU_PickAtlas software (http:// software.incf.org/software/wfu_pickatlas/ download). For each subject at a single time point (baseline and follow-up), the GMV in each ROI was computed by the sum of the volume of each voxel in the ROI. To illustrate the mean change in volume, we computed annualized percentage change by subtracting the baseline volume from the follow-up value, dividing by the baseline volume and multiplying by 100 .

\section{Correlation Analysis}

The Pearson correlation was performed to evaluate the relationship between the GMV in ROIs and clinical assessments in Table 1, including the Hoehn and Yahr stage, MDA-UPDRS III, Benton Judgment of Line Orientation score, Geriatric Depression Scale, MoCA, Semantic Fluency score, Hopkins Verbal Learning Test, Activities of Daily Living, Symbol Digit Modalities score, University of Pennsylvania Smell Identification Test, and Scales for Outcomes in Parkinson's disease-Autonomic. Two-tailed $P<.01$ was considered statistically significant.

\section{RESULTS}

\section{Demographic Characteristics}

No significant differences in sex, education level, and total intracranial volume were found between the patients with PD and controls. The MDSUPDRS III of PD was significantly higher than that of controls $(P<.001)$. Neuropsychological tests showed that behavioral deficits in the Geriatric Depression Scale $(P<.05)$ and cognitive deficits in the MoCA $(P<.05)$, semantic fluency total score $(P<$ $.05)$, Hopkins Verbal Learning Test Immediate Recall $(P<.05)$ and delayed recall $(P<.05)$, Symbol Digit Modalities score $(P<$ $.001)$, Smell Identification Test $(P<.001)$, and Scales for Outcomes in Parkinson's Disease-Autonomic $(P<.001)$ were more severe in the patients with PD than in the control group. In addition, a cognitive decline in the MoCA scores was found both for patients with PD $(P<.05)$ and controls $(P<.05)$ at follow-up compared with baseline. No significant differences in other cognitive subdomains (Benton Judgment of Line Orientation Score, Hopkins Verbal Learning Test Delayed Recognition False Alarms) were found between the 2 groups (Table 1).

\section{VBM Results}

The main effect of "group" revealed cortical and subcortical GM volume changes for patients with PD in contrast to controls. The 
decreased GM volume was found in the bilateral superior/middle frontal gyrus (Brodmann area [BA] 9/10), left inferior/middle temporal gyrus (BA 21/20/37), right superior/inferior parietal lobule (BA 7/40) and angular gyrus (BA 39), and the bilateral caudate body, while the increased GM volume was identified in the left inferior frontal gyrus (orbital, BA 47), right middle occipital gyrus (BA 19), right anterior cingulate cortex (anterior cingulate cortex, BA 32), bilateral globus pallidus inter-

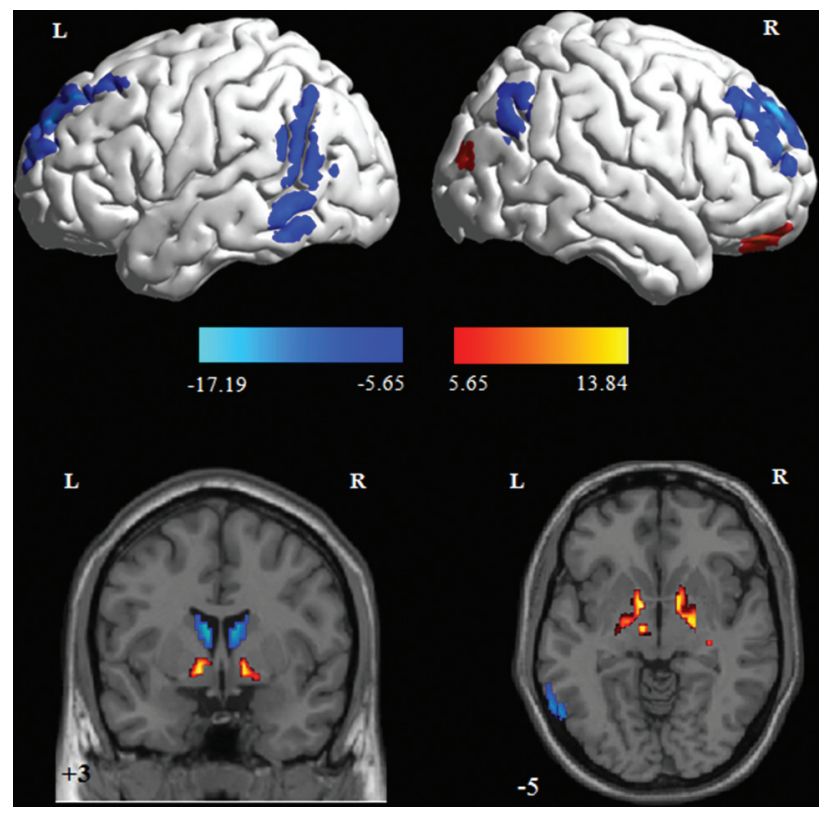

FIG 1. Gray matter volume changes between patients with PD and controls. The warm color indicates increased gray matter volume for patients with PD in contrast to controls, while the cool color indicates decreased gray matter volume for patients with PD in contrast to controls. Color bars indicate $t$ scores.

\section{Table 3: GMV changes with time}

\begin{tabular}{|c|c|c|c|c|c|}
\hline \multirow[b]{2}{*}{ Regions } & \multirow{2}{*}{$\begin{array}{c}\text { Cluster } \\
\text { Size } \\
\text { BA (Voxel) }\end{array}$} & \multicolumn{3}{|c|}{ MNI } & \multirow{2}{*}{$\begin{array}{c}T \\
\text { Score }\end{array}$} \\
\hline & & $x$ & $\mathbf{Y}$ & $\mathbf{Z}$ & \\
\hline \multicolumn{6}{|c|}{ Baseline vs follow-up: [(PD1 + Control1) $>$ (PD2 + Control2)] } \\
\hline Lt. caudate body & 566 & -8 & & 18 & 14.55 \\
\hline Lt. caudate body & & -6 & 11 & 13 & 14.24 \\
\hline Lt. caudate body & & -12 & -12 & 22 & 12.23 \\
\hline Rt. caudate body & 535 & 9 & 5 & 18 & 14.23 \\
\hline Rt. caudate body & & 12 & -3 & 22 & 13.17 \\
\hline Rt. caudate body & & 14 & -13 & 22 & 11.31 \\
\hline \multicolumn{6}{|c|}{$\begin{array}{l}\text { Follow-up vs baseline: }[(\mathrm{PD} 2+\text { Control } 2)>(\mathrm{PD1}+\text { Control } 1)] \\
\text { No significant activation }\end{array}$} \\
\hline \multicolumn{6}{|c|}{$\mathrm{PD1}>\mathrm{PD} 2$} \\
\hline Rt. caudate body & 192 & 8 & 9 & 15 & 8.27 \\
\hline Lt. caudate body & 151 & -6 & 12 & 10 & 7.92 \\
\hline \multicolumn{6}{|l|}{ Controll > Control2 } \\
\hline Lt. caudate body & 570 & -8 & & 18 & 16.85 \\
\hline Lt. caudate body & & -12 & -13 & 22 & 15.49 \\
\hline Lt. caudate body & & -17 & -21 & 24 & 7.37 \\
\hline Rt. caudate body & 552 & 12 & -3 & 22 & 15.21 \\
\hline Rt. caudate body & & 9 & 3 & 18 & 14.11 \\
\hline Rt. caudate body & & 14 & -15 & 22 & 14.09 \\
\hline$(\mathrm{PD} 2>$ Control2 $)>\left(\mathrm{PD} 1>\right.$ Control1 $^{\mathrm{a}}$ & & & & & \\
\hline Lt. thalamus & 160 & -21 & -24 & 3 & 5.79 \\
\hline$(\mathrm{PD} 1>$ Control 1$)>\left(\mathrm{PD} 2>\text { Control }^{2}\right)^{\mathrm{a}}$ & & & & & \\
\hline No significant activation & & & & & \\
\hline
\end{tabular}

nus, right putamen, left hippocampus, and left subthalamic nucleus (Table 2 and Fig 1).

The main effect of "time" revealed decreased GM volume in the bilateral caudate body for follow-up in contrast to baseline (Table 3 and Fig 2). In addition, the 2 simple main effects of time for patients with PD and controls also revealed decreased GM volume change in the bilateral caudate body with time (Table 3 ). Reduction in the caudate between baseline and 1-year follow-up was $1.96 \%$ for healthy controls and $1.1 \%$ for patients with PD. To compare with a previous study that observed volume reduction in the limbic/paralimbic regions for patients with $\mathrm{PD}$ with disease progression, we extracted the volumes in these regions. The results of paired $t$ tests showed that the decreased volumes in these regions did not reach significant differences.

In addition, we identified progressive GMV increase specific to patients with $\mathrm{PD}$ in the left thalamus with 160 voxels $(P<.05$ family-wise error corrected) (MNI coordinates: $-21,-24,3$ ) close to the ventral lateral nucleus by an interaction effect of "group" by "time" $[(\mathrm{PD} 2>\mathrm{PD} 1)>($ Control2 $>$ Control1 $)]$ (Fig 3).

\section{Correlation Results}

There were significant positive correlations between the caudate volume and the MoCA score for patients with PD and controls $(r=0.32, P=.0039$, and $r=0.38, P=.0047$, respectively; On-line Fig 1). There was no significant relationship between enlarged thalamic volume and MDS-UPDRS III scores. Twenty-three tremor-dominant patients with PD presented initially with tremor with relatively mild bradykinesia and rigidity, and the tremor scores were calculated on the basis of the summation of the rest tremor amplitude of the upper and lower limbs. However, the post hoc 2-tailed 2-sample $t$ test analysis showed that a positive relationship was found between the thalamic volume and the rest tremor score of 23 tremor-dominant patients with $\mathrm{PD}$ with scores ranging between 2 and 3 (On-line Fig 2).

To clarify the contributions of these measurements with significant differences between groups to the enlarged thalamic volume, we performed additional correlations, controlling these measurements as covariates of no interest. There was no significant correlation.

\section{DISCUSSION}

The aim of the present study was to investigate the GMV change in patients with PD and to explore the progressive GMV change specific to patients with PD with disease progression compared with controls. Reduced GMV was identified in the bilateral caudate for both patients with PD and healthy controls with time. It was revealed that patients with PD exhibited increased GMV in 

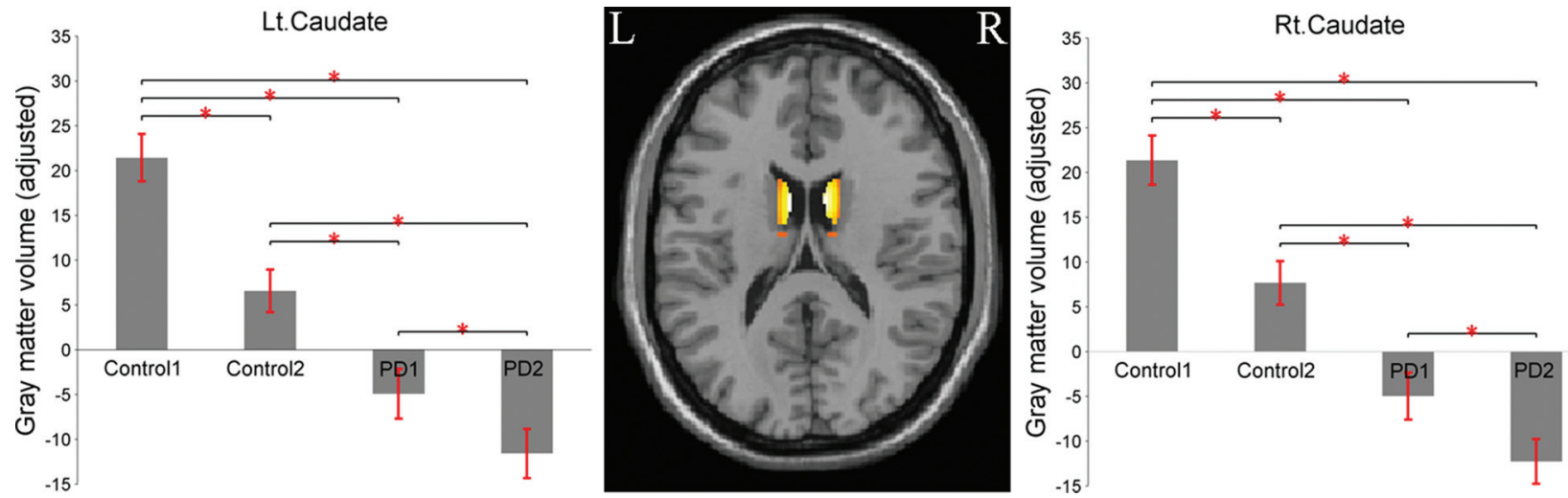

FIG 2. Barplot depicting the mean (and standard error) of the volume in the bilateral caudate between the 2 groups across time (adjusted for age, sex, years of education, imaging data from multiple centers, and total intracranial volume). The asterisk indicates significant differences at $P<.001$.
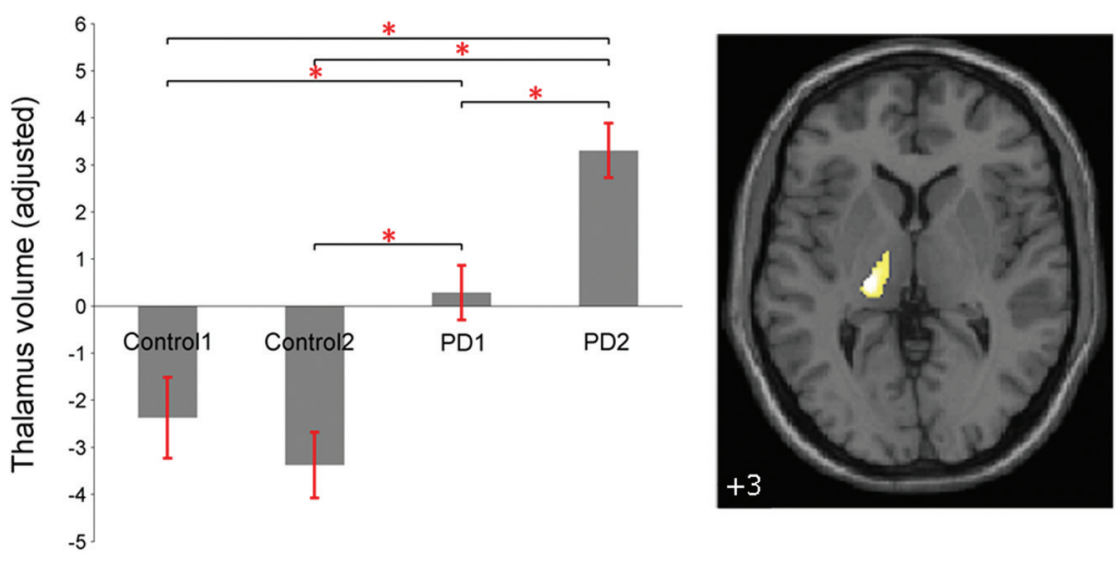

FIG 3. Barplot depicting the mean (and standard error) of the volume in the left thalamus specific to patients with PD across time (adjusted for age, sex, years of education, imaging data from multiple centers, and total intracranial volume). The asterisk indicates significant differences at $P<.001$.

the orbitofrontal gyrus, occipital cortex, limbic/paralimbic areas, and globus pallidus internus/putamen, whereas they had reduced GMV in the frontotemporoparietal network and the bilateral caudate, compared with controls. In addition, the progressive GMV change specific to patients with PD was found in the left thalamus.

\section{Group Differences: GMV Changes for Patients with PD Compared with Controls}

Previous studies have reported GMV loss in patients with PD with dementia (for a review, see Pan et $\mathrm{al}^{20}$ ). The present study extended the knowledge by showing that GMV loss can be found even in patients with early-stage PD. These regions included the frontal, temporal, and parietal regions and the subcortical area in the caudate bilaterally. The findings suggest that the observed GMV decreases may represent an early manifestation of underlying Parkinson disease-related pathologic changes. $^{6,7}$

However, the neuropathologic correlates of an increase in GMV are less clear. Consistent with previous studies, the present study observed the increased GMV in the limbic/paralimbic system and globus pallidus internus/putamen in patients with PD compared with controls. ${ }^{10,21,22}$ Although the present study pro- vides no evidence regarding the mechanisms leading to increased brain volume in $\mathrm{PD}$, as a chronically progressive neurodegenerative disorder, the effect may be due to a compensatory response to impaired cerebral function in early PD. ${ }^{10,21-24}$

In the present study, the findings of GMV changes (decrease and increase) in patients with PD were not wholly consistent with findings in any of the previous studies. The patient's heterogeneity (eg, age, sex, education, disease severity) may contribute to the inconsistencies in the pattern of findings among the studies. Further studies are required with a large sample size, controlling for the potential confounding effect.

\section{Longitudinal Evaluation: Progressive Caudate Volume Loss in Patients with PD and Controls}

In the present study, a significantly progressive decrease in the volume of the bilateral caudate was observed both in the patients with PD and controls. The reduction in the caudate was observed in several cross-sectional studies as part of the normal aging process. ${ }^{25-27}$ In the present study, the reduction of caudate volume was $1.96 \%$ /year for healthy controls and $1.1 \%$ /year for patients with PD. The reduction rates in the present study are similar to the ones reported in a 6-month longitudinal study, ${ }^{28}$ but greater than the ones reported in a 5-year longitudinal study. ${ }^{29}$ This heterogeneity may partially reflect differences among these populations. Additionally, follow-up intervals may contribute to differences in the reduction rate. In the present study, a reduction in the caudate was observed in patients with PD compared with controls both at baseline and follow-up, and this reduction remained in the comparison of baseline and control follow-up of patients with PD. Furthermore, a significant positive correlation was found between the MoCA score and the caudate volume for both patients with PD and healthy controls. The caudate is linked with the dorsolateral prefrontal cortex and lateral orbitofrontal cortex, and the dysfunction in this region is thought to contribute to the cog- 
nitive impairment in PD. ${ }^{30-33}$ The results suggest that the loss in caudate volume contributed to the cognitive decline for both groups with time, while the striatal dopaminergic deficiency in patients with PD may worsen the caudate volume loss compared with healthy controls.

A previous longitudinal study observed limbic/paralimbic loss including the hippocampus, hypothalamus, posterior cingulate cortex, and anterior cingulate cortex for patients with PD with disease progression with time. ${ }^{15}$ In contrast, we extracted the GMV in these regions, and the results showed that the decreased volumes did not reach a significant difference. The heterogeneity, methodologic differences, and sample size of patients between the 2 studies may have led to the discrepancies. In particular, the patients with PD in the present study had a shorter follow-up period (mean, 12 months) at an earlier disease stage (mean Hoehn and Yahr stage, $1.6 \pm 0.5$ ) than the patients in that study (mean follow-up period, 25 months; mean Hoehn and Yahr stage, $2.9 \pm$ 0.8 ) and a larger sample size ( 89 patients with PD) than in that study (13 patients with PD without dementia). In addition, no covariate was included in that study, though age, sex, years of education, and total intracranial volume may confound the results.

\section{Longitudinal Evaluation of Patients with PD versus Controls: Progressive Thalamic Volume Increase Specific to the Patients with PD}

In the present study, the progressive thalamic volume increase was observed specific to patients with PD compared with controls. On the contrary, the thalamic volume tended to decrease with time in controls. The thalamus occupies a pivotal position within the striatothalamocortical circuits, ${ }^{30,34,35}$ in which the thalamus receives input from the basal ganglia. ${ }^{36}$ In the present study, the enlarged thalamus close to the ventral lateral nucleus may relate to motor control. ${ }^{31,36}$ Consistent with previous studies, the positive correlation between the thalamic volume and the tremor score further suggests that the enlarged thalamic volume (mainly in the ventral lateral) may relate to tremor severity in PD. ${ }^{9,37}$

\section{CONCLUSIONS}

The present study investigated the progressive GMV change across time in patients with PD compared with controls. The observed progressive changes in gray matter volume in PD may provide new insights into the neurodegenerative process. The findings suggest that the caudate volume loss may contribute to cognitive decline in patients with PD and the progressive thalamic volume increase may relate to tremor severity in PD. However, the present study has the following 3 limitations: First, considering the highly nonlinear effect in age range, ${ }^{38}$ further study should be limited to a restricted sample with less age heterogeneity. Second, the brain volume change in the present study was seen only at 1-year follow-up, and further study should include multiple follow-up time windows to explore the dynamic changes of GM volume. Finally, to clarify whether the presence of tremor was actually driving the larger thalamic volumes, the study should compare thalamic volumes of tremor- and non-tremor-dominant patients with PD.
Disclosures: Xiuqin Jia_UNRELATED: Grants/Grants Pending: National Natural Science Foundation of China.

\section{REFERENCES}

1. Burton EJ, McKeith IG, Burn DJ, et al. Cerebral atrophy in Parkinson's disease with and without dementia: a comparison with Alzheimer's disease, dementia with Lewy bodies and controls. Brain 2004;127:791-800 CrossRef Medline

2. Beyer MK, Larsen JP, Aarsland D. Gray matter atrophy in Parkinson disease with dementia and dementia with Lewy bodies. Neurology 2007;69:747-54 CrossRef Medline

3. Kostic VS, Agosta F, Petrović I, et al. Regional patterns of brain tissue loss associated with depression in Parkinson disease. Neurology 2010;75:857-63 CrossRef Medline

4. Kostic VS, Agosta F, Pievani M, et al. Pattern of brain tissue loss associated with freezing of gait in Parkinson disease. Neurology 2012;78:409-16 CrossRef Medline

5. Compta Y, Ibarretxe-Bilbao N, Pereira J, et al. Grey matter volume correlates of cerebrospinal markers of Alzheimer-pathology in Parkinson's disease and related dementia. Parkinsonism Relat Disord 2012;18:941-47 CrossRef Medline

6. Hong J, Lee JE, Sohn YH, et al. Neurocognitive and atrophic patterns in Parkinson's disease based on subjective memory complaints. J Neurol 2012;259:1706-12 CrossRef Medline

7. Lee EY, Sen S, Eslinger PJ, et al. Early cortical gray matter loss and cognitive correlates in non-demented Parkinson's patients. Parkinsonism Relat Disord 2013;19:1088-93 CrossRef Medline

8. Lee JE, Cho $\mathrm{KH}, \mathrm{Ham} \mathrm{JH}$, et al. Olfactory performance acts as a cognitive reserve in non-demented patients with Parkinson's disease. Parkinsonism Relat Disord 2014;20:186-91 CrossRef Medline

9. Kassubek J, Juengling FD, Hellwig B, et al. Thalamic gray matter changes in unilateral Parkinsonian resting tremor: a voxel-based morphometric analysis of 3-dimensional magnetic resonance imaging. Neurosci Lett 2002;323:29-32 CrossRef Medline

10. Pagonabarraga J, Soriano-Mas C, Llebaria G, et al. Neural correlates of minor hallucinations in non-demented patients with Parkinson's disease. Parkinsonism Relat Disord 2014;20:290-96 CrossRef Medline

11. Nagano-Saito A, Washimi Y, Arahata Y, et al. Cerebral atrophy and its relation to cognitive impairment in Parkinson disease. Neurology 2005;64:224-29 CrossRef Medline

12. Ibarretxe-Bilbao N, Ramirez-Ruiz B, Tolosa E, et al. Hippocampal head atrophy predominance in Parkinson's disease with hallucinations and with dementia. J Neurol 2008;255:1324-31 CrossRef Medline

13. Tessitore A, Amboni M, Cirillo G, et al. Regional gray matter atrophy in patients with Parkinson disease and freezing of gait. AJNR Am J Neuroradiol 2012;33:1804-09 CrossRef Medline

14. Ellfolk U, Joutsa J, Rinne JO, et al. Striatal volume is related to phonemic verbal fluency but not to semantic or alternating verbal fluency in early Parkinson's disease. J Neural Transm 2014;121:33-40 CrossRef Medline

15. Ramirez-Ruiz B, Marti MJ, Tolosa E, et al. Longitudinal evaluation of cerebral morphological changes in Parkinson's disease with and without dementia. J Neurol 2005;252:1345-52 CrossRef Medline

16. Parkinson Progression Marker Initiative. The Parkinson Progression Marker Initiative (PPMI). Prog Neurobiol 2011;95:629-35 CrossRef Medline

17. Yesavage JA. Geriatric Depression Scale. Psychopharmacol Bull 1988; 24:709-11 Medline

18. Ashburner J. A fast diffeomorphic image registration algorithm. Neuroimage 2007;38:95-113 CrossRef Medline

19. Good CD, Johnsrude IS, Ashburner J, et al. A voxel-based morphometric study of ageing in $\mathbf{4 6 5}$ normal adult human brains. Neuroimage 2001;14:21-36 CrossRef Medline

20. Pan P, Shi H, Zhong J, et al. Gray matter atrophy in Parkinson's disease with dementia: evidence from meta-analysis of voxel-based morphometry studies. Neurol Sci 2013;34:613-19 CrossRef Medline 
21. Binkofski F, Reetz, Gaser C, et al. Morphometric fingerprint of asymptomatic Parkin and PINK1 mutation carriers in the basal ganglia. Neurology 2007;69:842-50 CrossRef Medline

22. Krabbe K, Karlsborg M, Hansen A, et al. Increased intracranial volume in Parkinson's disease. J Neurol Sci 2005;239:45-52 CrossRef Medline

23. Helmich RC, de Lange FP, Bloem BR, et al. Cerebral compensation during motor imagery in Parkinson's disease. Neuropsychologia 2007;45:2201-15 CrossRef Medline

24. Palop JJ, Chin J, Mucke L. A network dysfunction perspective on neurodegenerative diseases. Nature 2006;443:768-73 CrossRef Medline

25. Jernigan TL, Archibald SL, Berhow MT, et al. Cerebral structure on MRI, Part I: localization of age-related changes. Biol Psychiatry 1991;29:55-67 CrossRef Medline

26. Walhovd KB, Fjell AM, Reinvang I, et al. Effects of age on volumes of cortex, white matter and subcortical structures. Neurobiol Aging 2005;26:1261-70; discussion 1275-78 CrossRef Medline

27. Abedelahi A, Hasanzadeh $\mathrm{H}, \mathrm{Hadizadeh} \mathrm{H}$, et al. Morphometric and volumetric study of caudate and putamen nuclei in normal individuals by MRI: effect of normal aging, gender and hemispheric differences. Pol J Radiol 2013;78:7-14 CrossRef Medline

28. Raz N, Schmiedek F, Rodrigue KM, et al. Differential brain shrinkage over 6 months shows limited association with cognitive practice. Brain Cogn 2013;82:171-80 CrossRef Medline

29. Raz N, Lindenberger U, Rodrigue KM, et al. Regional brain changes in aging healthy adults: general trends, individual differences and modifiers. Cerebral Cortex 2005;15:1676-89 CrossRef Medline
30. Alexander GE, DeLong MR, Strick PL. Parallel organization of functionally segregated circuits linking basal ganglia and cortex. Ann Rev Neurosci 1986;9:357-81 CrossRef Medline

31. Poston KL, Eidelberg D. Functional brain networks and abnormal connectivity in the movement disorders. Neuroimage 2012;62: 2261-70 CrossRef Medline

32. Jokinen $P$, Brück A, Aalto $S$, et al. Impaired cognitive performance in Parkinson's disease is related to caudate dopaminergic hypofunction and hippocampal atrophy. Parkinsonism Relat Disord 2009;15: 88-93 CrossRef Medline

33. Rinne JO, Portin R, Ruottinen H, et al. Cognitve impairment and the brain dopaminergic system in Parkinson disease: [F18] fluorodopa positron emission tomographic study. Arch Neurol 2000;57:470-75 CrossRef Medline

34. Albin RL, Young AB, Penney JB. The functional anatomy of basal ganglia disorders. Trends Neurosci 1989;12:366-75 CrossRef Medline

35. Evarts EV, Kimura M, Wurtz RH, et al. Behavioral correlates of activity in basal ganglia neurons. Trends in Neurosci 1984;7:447-53 CrossRef

36. DeLong MR, Wichmann T. Circuits and circuit disorders of the basal ganglia. Arch Neurol 2007;64:20-24 CrossRef Medline

37. Halliday GM. Thalamic changes in Parkinson's disease. Parkinsonism Relat Disord 2009;15:S152-S155 CrossRef

38. Fjell AM, Westlye LT, Grydeland H, et al; Alzheimer Disease Neuroimaging Initiative. Critical ages in the life course of the adult brain: nonlinear subcortical aging. Neurobiol Aging 2013;34:2239-47 CrossRef Medline 